\title{
IMPLEMENTAÇÃO DA TÉCNICA DE EXTRAÇÃO DE DISPERSÃO DA MATRIZ EM FASE SÓLIDA ("MSPD”) PARA DETERMINAÇÃo DE RESÍDUOS DE AGROTÓXICOS EM LARANJAS'
}

\author{
Maria Helena W. M. CARDOSO,*, Lúcia Helena P. BASTOS ${ }^{2}$, Tatiane S. NEVES ${ }^{2}$, Shirley ABRANTES ${ }^{2}$
}

\begin{abstract}
RESUMO
Um método de extração de multiresíduos baseado na técnica de dispersão da matriz em fase sólida ("MSPD”), foi otimizado e validado para a extração e análise cromatográfica de 27 agrotóxicos (isômeros $\alpha, \beta, \gamma, \delta$ do hexaclorociclohexano (HCH), dieldrin, endrin, heptacloro e seu epóxido (HE), $\alpha$ e $\beta$-endosulfan, $o, p^{\prime}$-DDD, $p, p^{\prime}$-DDD, $o, p^{\prime}$-DDE, $p, p^{\prime}$-DDE, $o, p^{\prime}$-DDT, $p, p^{\prime}$-DDT, dicofol, metoxicloro, mirex, hexaclorobenzeno (HCB), clorotalonil, parationa metilica, fenitrotiona, malationa, folpete, diazinona, cis e trans-permetrina em laranjas. A amostra macerada é inicialmente homogeneizada com $\mathrm{C}_{18}$, o homogeneizado é transferido para uma coluna de vidro contendo sílica gel onde se adiciona $10 \mathrm{~mL}$ de acetato de etila como solvente de eluição. O eluente é concentrado, diluído em isooctano e $1 \mu \mathrm{L}$ deste é injetado no cromatógrafo a gás. Para a separação e quantificação dos 27 agrotóxicos, foi utilizado um CG/DCE. A confirmação dos mesmos foi feita por espectrometria de massas. Para a quantificação dos 27 agrotóxicos utilizou-se a padronização externa. As recuperações dos mesmos variaram de 70 a 120\%, considerando-se os níveis de adição agrotóxicos/amostra de 0,02 e $2,0 \mathrm{mg} / \mathrm{kg}$. Os limites de quantificação variaram de 0,01 a $0,5 \mathrm{mg} / \mathrm{kg}$.

Palavras-chave: agrotóxicos; laranjas; cromatografia em fase gasosa; dispersão da matriz em fase sólida ("MSPD").
\end{abstract}

\section{SUMMARY}

IMPLEMENTATION OF MSPD TECHNIQUE TO DETERMINATION OF PESTICIDES RESIDUES IN ORANGES. A multiresidue method of extraction based on matrix solid-phase dispersion (MSPD) technique was optimized and validated for chromatographic analyses of twenty seven pesticides residues [hexachlorocyclohexane $(\mathrm{HCH})$ isomers $(\alpha, \beta, \gamma, \delta)$, dieldrin, endrin, heptachlor and its epoxide (HE), $\alpha$ and $\beta$-endosulfan, o,p'-DDD, p,p'-DDD, o,p'-DDE, p,p'-DDE, o,p'-DDT p,p'-DDT, dicofol, methoxychlor, mirex, hexachlorobenzene (HCB), chlorotalonil, parathion methyl, fenitrothion, malathion, folpet, diazinon, permethrin (cis and trans) in oranges. The sample was blended with $\mathrm{C}_{18}$ and then the homogenized sample was introduced onto a glass column containing silica. Ten mL of ethyl acetate was added to the column as elution solvent. The eluent was concentrated, diluted in isooctane and $1 \mu \mathrm{L}$ was injected into the gas chromatograph. The analyses of pesticides were performed using a GC/ECD. Confirmation was done by mass spectrometry. The recovery ranged from 70 to $120 \%$ in the concentration range of 0.02 to $2.0 \mathrm{mg} / \mathrm{kg}$. The limits of quantification ranged from 0.01 to $0.5 \mathrm{mg} / \mathrm{kg}$.

Keywords: pesticides; oranges; gas chromatography; matrix solid phase dispersion.

\section{1 - INTRODUÇÃO}

Os citros são as frutas mais cultivadas em todo o mundo. Entre as variedades cítricas, a laranja é a principal representante do grupo.

A cadeia de citros contempla as frutas in natura, a produção de sucos (concentrados, reconstituídos, pasteurizados e frescos) e de óleos essenciais e "pellets" de polpa seca - os dois últimos voltados ao uso industrial [8].

O Brasil está entre os quatro principais países produtores, onde $2 \%$ da área cultivada é coberta por laranjais, que se espalham por mais de 27 mil estabelecimentos rurais [8].

A laranja é a fruta que mais se destaca no cenário brasileiro, detendo $30 \%$ da produção mundial, de acordo com dados da safra de 1998/1999 [7]. No Brasil, a produção obtida na safra de 2002 alcançou aproxima-

\footnotetext{
Recebido para publicação em 01/10/2003. Aceito para publicação em 26/01/2004 (001217).

2. Laboratório de Alimentos e Contaminantes, Departamento de Quimica, I.N.C.Q.S. / Fundação Oswaldo Cruz, Avenida Brasil, 4365-Manguinhos, Rio de Janeiro-RJ, CEP: 21.045-900.E-mail: helenawohlers@hotmail.com * A quem a correspondência deve ser enviada.
}

damente 18,5 milhões de toneladas de acordo com dados do IBGE [6] e FAO [5]. Os maiores produtores de laranja do país são os Estados de São Paulo, Bahia e Sergipe sendo as principais variedades cultivadas: a Pêra, a Natal, a Hamlin e a Lima.

A laranja produzida no país é destinada principalmente à indústria de sucos e, cerca de $10 \%$ da produção de laranja vai para a venda in natura. Com a produção do suco de laranja industrializado o Brasil torna-se um grande exportador, cerca de $70 \%$ do total, e a União Européia a principal importadora. Para o mercado americano, seguem outros $20 \%$ - participação que veio caindo ao longo da década passada por causa da concorrência dos mexicanos. Todo produto agrícola exportado é submetido a normas de controle que prevêem, entre outras coisas, a realização de análise de resíduos de agrotóxicos. Um dos grandes problemas que atingem a produção de laranjas é o descuido com a fitossanidade $[2,5,7,8]$, este fato leva a necessidade do controle da qualidade das laranjas no que diz respeito a agrotóxicos já que compromete a qualidade do fruto inviabilizando seu consumo e comercialização, além de trazer prejuízos econômicos para o produtor.

Os métodos tradicionais para análise de resíduos de agrotóxicos com extração por solventes são normalmente muito trabalhosos e envolvem um tempo de preparo e extração da amostra demasiadamente longo. 
O objetivo deste trabalho foi estudar, otimizar e validar simultaneamente a extração e a determinação de 27 resíduos de agrotóxicos, isômeros $(\alpha, \beta, \gamma, \delta)$ do hexaclorociclohexano $(\mathrm{HCH})$, dieldrin, endrin, heptacloro e seu epóxido (HE), hexaclorobenzeno (HCB), $\alpha$ e $\beta$ endosulfan, p,p'-DDD, o,p'-DDE, p,p'-DDE, o,p'-DDT, p,p'-DDT, dicofol, metoxicloro, mirex, clorotalonil, parationa metílica, fenitrotiona, folpete, malationa, diazinona, cis e trans-permetrina em laranjas Lima (casca + polpa - laranja inteira, polpa e casca separadamente) utilizando a cromatografia gasosa de alta resolução acoplada a detector por captura de elétrons (CGAR-DCE). Buscando otimizar as análises e diminuir o tempo de preparo de amostra foi experimentada neste trabalho a técnica de extração dos resíduos da dispersão da matriz em fase sólida ("MSPD"), baseada em trabalhos de TORRES, PICÓ \& MAÑES [11] e TORRES et al. [12], com algumas pequenas modificações, proporcionando rapidez e simplicidade no procedimento analítico. Dentre os agrotóxicos estudados apenas o dicofol, a diazinona, o clorotalonil e o folpete são permitidos na cultura de citros no Brasil nos níveis de 5mg/kg; 0,7mg/kg; 0,5mg/kg e $10,0 \mathrm{mg} / \mathrm{kg}$, respectivamente [10]. Vale salientar que outros resíduos podem ser encontrados em laranjas devido a uma aplicação indevida ou mesmo uma contaminação do meio ambiente e, sendo assim, sua pesquisa não deve ser descartada.

\section{2 - MATERIAL E MÉTODOS}

\section{1 - Amostras}

As amostras de laranja Lima testadas e analisadas foram compradas em um mercado de produtos orgânicos, no Rio de Janeiro, com a finalidade de se obter uma amostra testemunho sem os agrotóxicos estudados. Obteve-se um total de amostras de laranjas de três produtores diferentes. A região produtora localiza-se no município de Itaboraí, no Estado do Rio de Janeiro.

As amostras foram cortadas com faca em pequenos pedaços e moídas inteiras (casca + polpa) e individualmente a polpa e a casca com a finalidade de se realizar uma avaliação de cada parte da laranja, já que a maior parte dos agrotóxicos podem ficar retidos na casca.

\section{2 - Padrões e reagentes}

Foram utilizados padrões de agrotóxicos de pureza superior a 95\% (Dr. Ehrenstorfer), acetato de etila e isooctano grau resíduos de pesticidas (Merck).

Fase para SPE de $\mathrm{C}_{18}$ (Alltech), sílica gel 60 (Aldrich), coluna de vidro vazia para SPE com capacidade para $8 \mathrm{~mL}$ (J. T. Baker).

Soluções de trabalho contendo os 27 agrotóxicos estudados nas concentrações de: 0,$01 ; 0,02 ; 0,1 ; 0,2$; 0,$4 ; 0,6 ; 0,8$ e $1,0 \mu \mathrm{g} / \mathrm{mL}$ foram preparadas em isooctano a partir de soluções estoque de cada substância na concentração de $100,00 \mu \mathrm{g} / \mathrm{mL}$, com o objetivo de encontrar na amostra concentrações na faixa de 0,02 a $2,0 \mathrm{mg} / \mathrm{kg}$. A faixa de trabalho estabelecida está de acor- do com a sensibilidade e o LMR (limite máximo de resíduo permitido) dos agrotóxicos avaliados.

\section{3 - Procedimento analítico para a extração por "MSPD"}

Foram pesados $0,5 \mathrm{~g}$ da amostra moída em um béquer, adicionados $0,5 \mathrm{~g}$ de $\mathrm{C}_{18}$ e homogeneizados com bastão de vidro. Transferiu-se a amostra para a coluna, de SPE (contendo 0,5g de sílica gel 60) e adicionado $10 \mathrm{~mL}$ de acetato de etila a mesma, deixando o solvente eluir sob leve vácuo. O eluato foi recolhido em tubo de ensaio e, em seguida foi concentrado à secura sob leve corrente de nitrogênio. O resíduo foi diluído em $2 \mathrm{~mL}$ de isooctano, transferido para um "vial" e $1 \mu l$ do extrato foi injetado no cromatógrafo $[4,11,12]$.

\section{4 - Condições cromatográficas}

Cromatógrafo a gás HP 6890 (Agilent), equipado com detector por captura de elétrons, sistema de injeção automático e estação de trabalho - ChemStation. Temperatura do injetor e detector de $210^{\circ} \mathrm{C}$ e $300^{\circ} \mathrm{C}$, respectivamente. Coluna 5\% fenil metil siloxano - HP 5MS de $30 \mathrm{~m}$ de comprimento; $0,25 \mathrm{~mm}$ de diâmetro interno e $0,25 \mu \mathrm{m}$ de espessura de filme, nas temperaturas de $80^{\circ} \mathrm{C}$ $(0 \mathrm{~min}){ }^{\circledR 30^{\circ} \mathrm{C} / \mathrm{min}} 180^{\circ} \mathrm{C}(8 \mathrm{~min}){ }^{\circledR 2^{\circ} \mathrm{C} / \mathrm{min}} 200^{\circ} \mathrm{C}(5 \mathrm{~min}){ }^{\circledR 6^{\circ} \mathrm{C} / \mathrm{min}}$ $280^{\circ} \mathrm{C}$ (5 min). Fluxo de gás carreador (Nitrogênio) $=$ $1,7 \mathrm{~mL} / \mathrm{min}$, fluxo da purga do septo $=60,1 \mathrm{~mL} / \mathrm{min}$, fluxo total $=64,1 \mathrm{~mL} / \mathrm{min}$, fluxo do gás "make-up"(Nitrogênio) = $60 \mathrm{~mL} / \mathrm{min}$, injeção "splitless" = 0,75min.

\section{5 - Confirmação por espectrometria de massas}

A análise qualitativa das três amostras de laranja Lima orgânica foram realizadas no Laboratório de Toxicologia do ITEP-PE, segundo ARAÚJO et al. [1].

\section{6 - Estudo do tempo ideal de contato em amos- tra fortificada de laranja}

Para a validação de um procedimento analítico vários parâmetros são necessários para demonstrar que o mesmo é adequado para a aplicação pretendida. Entre os parâmetros a taxa de recuperação de um método é de fundamental importância já que busca demonstrar a relação existente entre a quantidade do analito a ser analisado, obtido após seguir o procedimento analítico completo, e a quantidade adicionada no início do procedimento analítico, ou seja, a exatidão do método. Na literatura são relatados diferentes tempos de contato entre o intervalo de fortificação da amostra e a extração propriamente dita [11, 12].

Visando uma maior taxa de recuperação em um menor tempo de contato possível, realizou-se um estudo mantendo duplicatas das amostras de polpa de laranja fortificadas com os 27 agrotóxicos na concentração de $0,2 \mu \mathrm{g} / \mathrm{mL}$, por períodos que variaram de 1 a 5 horas antes do início do procedimento indicado em 2.3. Esse intervalo de tempo foi estipulado para permitir que o procedimento completo fosse realizado no mesmo dia. Os resultados demonstraram claramente para 
a grande maioria dos agrotóxicos ser de 3 horas o tempo ideal de contato.

\section{7 - Validação da metodologia}

A validação do método foi conduzida segundo o procedimento de validação do Setor de Resíduos de Pesticidas do INCQS/FIOCRUZ [9].

a) No estudo da recuperação dos agrotóxicos da amostra de laranja lima, $1 \mathrm{~mL}$ da solução de trabalho, de 8 diferentes níveis, foram adicionados a $0,5 \mathrm{~g}$ da amostra picada (casca + polpa, a polpa e a casca). Depois disso, foram misturados com bastão de vidro visando garantir sua homogeneidade. As amostras fortificadas, preparadas em triplicatas de cada nivel de concentração, foram mantidas por 3 horas a temperatura ambiente antes do procedimento de extração (2.3), para evaporação do solvente.

b) As concentrações finais dos pesticidas na amostra foram de 0,$02 ; 0,04 ; 0,2 ; 0,4 ; 0,8 ; 1,2 ; 1,6 \mathrm{e}$ $2,0 \mathrm{mg} / \mathrm{kg}$. Essas concentrações foram usadas para elaboração da curva de calibração e cálculos dos limites de detecção (LD), limite de quantificação (LQ), coeficiente de variação (CV) ou desvio padrão relativo (SR\%), (a precisão), e recuperação (a exatidão) para o método estudado utilizando-se do método da padronização externa para quantificação dos analitos. Para a avaliação da exatidão e precisão foram adotados como referência os valores estabelecidos pelo CODEX ALIMENTARIUS [3].

\section{3 - RESULTADOS E DISCUSSÃO}

A amostra de laranja Lima orgânica testemunha apresentou-se satisfatória para avaliação do método realizado, já que não continha os agrotóxicos estudados, porém em estudo qualitativo, da casca + polpa, foi possivel a identificação do agrotóxico organofosforado metidationa que apresenta um LMR de $2,0 \mathrm{mg} / \mathrm{kg}$ para citros. A presença da metidationa foi confirmada, por análise por espectrometria de massas, nas três amostras de laranja Lima orgânica avaliadas.

De acordo com o estudo do tempo ideal de contato de fortificação das amostras o período de três horas mostrou-se adequado (Tabela 1), embora para a malationa, o dicofol, a fenitrotiona, o clorotalonil, o folpete e a parationa metílica, a recuperação tenha sido superior para o intervalo de tempo de 4 horas.

Na Figura 1 são apresentados os cromatogramas do branco da amostra (amostra testemunho) referente à casca + polpa, a polpa e a casca, respectivamente e da mistura padrão. Os picos que apareceram no branco da amostra não coincidem com os dos 27 agrotóxicos em questão, não interferindo na análise com as condições cromatográficas determinadas. O perfil cromatográfico da amostra da polpa se apresenta mais limpo, com menos interferentes, porém o CODEX ALIMENTARIUS [3] estabelece que na laranja a determinação analítica seja feita na amostra inteira (casca + polpa).
TABELA 1. Tempo ideal de contato de amostras fortificadas antes de procedimento de extração por "MSPD"

\begin{tabular}{|c|c|c|c|c|c|}
\hline Agrotóxico & $\begin{array}{c}\text { Tempo de } \\
\text { Contato - 1h } \\
\text { (recuperação-\%) }\end{array}$ & $\begin{array}{c}\text { Tempo de } \\
\text { Contato - } 2 \mathrm{~h} \\
\text { (recuperação-\%) }\end{array}$ & $\begin{array}{c}\text { Tempo de } \\
\text { Contato - 3h } \\
\text { (recuperação-\%) }\end{array}$ & $\begin{array}{c}\text { Tempo de } \\
\text { Contato - 4h } \\
\text { (recuperação-\%) }\end{array}$ & $\begin{array}{c}\text { Tempo de } \\
\text { Contato-5h } \\
\text { (recuperação-\% }\end{array}$ \\
\hline$\alpha-\mathrm{HCH}$ & 62 & 62 & 71 & 67 & 61 \\
\hline $\mathrm{HCB}$ & 61 & 62 & 73 & 67 & 60 \\
\hline$\beta-\mathrm{HCH}$ & 73 & 75 & 78 & 73 & 71 \\
\hline$\gamma \mathrm{HCH}$ & 65 & 66 & 73 & 69 & 65 \\
\hline$\delta \mathrm{HCH}$ & 84 & 85 & 89 & 87 & 84 \\
\hline heptacloro & 69 & 70 & 81 & 71 & 69 \\
\hline malationa & 67 & 70 & 72 & 77 & 70 \\
\hline dicofol & 44 & 44 & 45 & 63 & 62 \\
\hline $\mathrm{HE}$ & 74 & 75 & 81 & 76 & 73 \\
\hline$o, p^{\prime}-\mathrm{DDE}$ & 79 & 79 & 85 & 81 & 79 \\
\hline$\alpha$-endosulfan & 73 & 74 & 79 & 76 & 73 \\
\hline$p, p^{\prime}$-DDE & 68 & 69 & 75 & 70 & 66 \\
\hline dieldrin & 72 & 72 & 77 & 72 & 70 \\
\hline endrin & 79 & 80 & 85 & 82 & 79 \\
\hline$\beta$-endosulfan & 74 & 75 & 79 & 77 & 73 \\
\hline$p, p^{\prime}-\mathrm{DDD}$ & 77 & 78 & 83 & 78 & 75 \\
\hline$o, p^{\prime}$-DDT & 80 & 81 & 86 & 83 & 81 \\
\hline$p, p^{\prime}$-DDT & 82 & 84 & 87 & 85 & 82 \\
\hline metoxicloro & 80 & 83 & 86 & 84 & 79 \\
\hline diazinona & 68 & 71 & 78 & 74 & 72 \\
\hline fenitrotiona & 61 & 64 & 64 & 72 & 63 \\
\hline clorotalonil & 65 & 65 & 66 & 73 & 64 \\
\hline permetrina-cis & 80 & 80 & 85 & 80 & 78 \\
\hline folpete & 107 & 106 & 107 & 124 & 110 \\
\hline $\begin{array}{l}\text { parationa } \\
\text { metílica }\end{array}$ & 64 & 66 & 66 & 75 & 64 \\
\hline $\begin{array}{l}\text { permetrina- } \\
\text { trans }\end{array}$ & 91 & 83 & 86 & 76 & 83 \\
\hline mirex & 75 & 75 & 80 & 77 & 76 \\
\hline
\end{tabular}

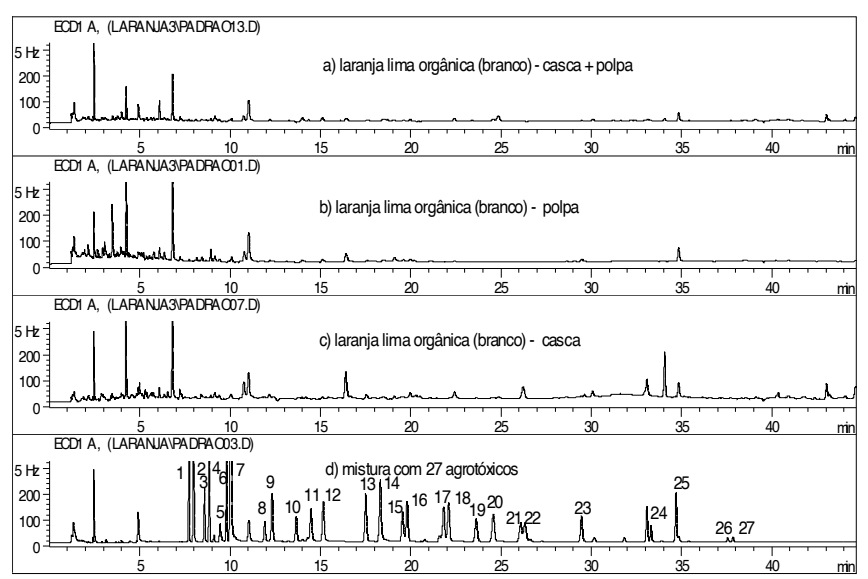

FIGURA 1. Cromatogramas obtidos da amostra de laranja Lima orgânica (amostra testemunho) nas condições especificadas anteriormente para: (a) $1 \mu \mathrm{L}$ da casca + polpa, (b) $1 \mu \mathrm{L}$ da polpa, (c) $1 \mu \mathrm{L}$ da casca e (d) $1 \mu \mathrm{L}$ da mistura com 27 agrotóxicos na faixa de concentração entre $0,05 \mu \mathrm{g} / \mathrm{mL}$ a $0,3 \mu \mathrm{g} / \mathrm{mL}$ de (1) $\alpha-\mathrm{HCH}$, (2) $\mathrm{HCB}$, (3) $\beta-\mathrm{HCH}$, (4) $\gamma-\mathrm{HCH}$, (5) diazinona, (6) $\delta$ - $\mathrm{HCH},(7)$ clorotalonil, (8) parationa metílica, (9) heptacloro, (10) fenitrotiona, (11) malationa, (12) dicofol, (13) HE, (14) folpete, (15) o,p'DDE, (16) $\alpha$-endosulfan, (17) $p, p$-DDE, (18) dieldrin, (19) endrin, (20) $\beta$-endosulfan, (21) $p$,p'-DDD, (22) o,p'-DDT, (23) p,p'-DDT, (24) metoxicloro, (25) mirex, (26) cispermetrina, (27) trans-permetrina. 
TABELA 2. Limites de detecção (LD) e de quantificação (LQ) calculados para a laranja Lima (casca + polpa; polpa e casca)

\begin{tabular}{|c|c|c|c|c|c|c|}
\hline \multirow[b]{2}{*}{ Agrotóxico } & \multicolumn{2}{|c|}{ Casca + Polpa ${ }^{(a)}$} & \multicolumn{2}{|c|}{ Polpa $^{(a)}$} & \multicolumn{2}{|c|}{ Casca $^{(a)}$} \\
\hline & $\begin{array}{c}\text { LD } \\
(\mathrm{mg} / \mathrm{kg})\end{array}$ & $\begin{array}{c}\mathrm{LQ} \\
(\mathrm{mg} / \mathrm{kg})\end{array}$ & $\begin{array}{c}\text { LD } \\
(\mathrm{mg} / \mathrm{kg})\end{array}$ & $\begin{array}{c}\mathrm{LQ} \\
(\mathrm{mg} / \mathrm{kg})\end{array}$ & $\begin{array}{c}\text { LD } \\
(\mathrm{mg} / \mathrm{kg})\end{array}$ & $\begin{array}{c}\mathrm{LQ} \\
(\mathrm{mg} / \mathrm{kg})\end{array}$ \\
\hline$\alpha-\mathrm{HCH}$ & 0,05 & 0,15 & 0,08 & 0,23 & 0,07 & 0,23 \\
\hline $\mathrm{HCB}$ & 0,08 & 0,26 & 0,13 & 0,40 & 0,10 & 0,32 \\
\hline$\beta-\mathrm{HCH}$ & 0,06 & 0,20 & 0,06 & 0,20 & 0,10 & 0,30 \\
\hline$\gamma \mathrm{HCH}$ & 0,05 & 0,17 & 0,06 & 0,20 & 0,08 & 0,24 \\
\hline$\delta-\mathrm{HCH}$ & 0,04 & 0,11 & 0,04 & 0,13 & 0,06 & 0,20 \\
\hline heptacloro & 0,06 & 0,18 & 0,04 & 0,13 & 0,09 & 0,30 \\
\hline malationa & 0,10 & 0,30 & 0,16 & 0,46 & 0,18 & 0,56 \\
\hline dicofol & 0,16 & 0,48 & 0,10 & 0,30 & 0,07 & 0,21 \\
\hline $\mathrm{HE}$ & 0,05 & 0,16 & 0,05 & 0,15 & 0,07 & 0,22 \\
\hline $0, p^{\prime}-\mathrm{DDE}$ & 0,05 & 0,17 & 0,06 & 0,19 & 0,10 & 0,30 \\
\hline$\alpha$-endosulfan & 0,04 & 0,14 & 0,05 & 0,16 & 0,08 & 0,30 \\
\hline$p, p^{\prime}-\mathrm{DDE}$ & 0,04 & 0,12 & 0,03 & 0,10 & 0,06 & 0,20 \\
\hline dieldrin & 0,04 & 0,12 & 0,05 & 0,16 & 0,06 & 0,20 \\
\hline endrin & 0,04 & 0,12 & 0,04 & 0,13 & 0,06 & 0,20 \\
\hline$\beta$-endosulfan & 0,04 & 0,11 & 0,05 & 0,15 & 0,07 & 0,20 \\
\hline$p, p^{\prime}$-DDD & 0,04 & 0,13 & 0,05 & 0,15 & 0,06 & 0,20 \\
\hline$o, p^{\prime}$-DDT & 0,05 & 0,16 & 0,06 & 0,18 & 0,08 & 0,26 \\
\hline$p, p^{\prime}$-DDT & 0,04 & 0,13 & 0,04 & 0,12 & 0,07 & 0,20 \\
\hline metoxicloro & 0,07 & 0,20 & 0,07 & 0,20 & 0,06 & 0,20 \\
\hline diazinona & 0,13 & 0,38 & 0,16 & 0,50 & 0,10 & 0,30 \\
\hline fenitrotiona & 0,06 & 0,18 & 0,06 & 0,19 & 0,08 & 0,20 \\
\hline clorotalonil & 0,10 & 0,30 & 0,11 & 0,34 & 0,07 & 0,20 \\
\hline permetrina-cis & 0,08 & 0,24 & 0,10 & 0,30 & 0,10 & 0,30 \\
\hline folpete & 0,13 & 0,40 & 0,06 & 0,17 & 0,20 & 0,60 \\
\hline parationa metílica & 0,04 & 0,14 & 0,06 & 0,18 & 0,10 & 0,30 \\
\hline permetrina-trans & 0,06 & 0,19 & 0,09 & 0,26 & 0,10 & 0,40 \\
\hline mirex & 0,06 & 0,18 & 0,09 & 0,26 & 0,11 & 0,30 \\
\hline
\end{tabular}

(a) Dados foram obtidos através da média de três determinações realizadas em paralelo para cada nível de concentração estudado.
Na Tabela 2 são apresentados os LD's e os LQ's calculados para a amostra de laranja Lima. Os valores dos LD's e dos LQ's foram calculados utilizando os valores do desvio padrão dos resíduos e do coeficiente angular da curva de calibração. Os LD's e os LQ's calculados para o dicofol, o clorotalonil, a diazinona e o folpete estão inferiores ao LMR estabelecido para cada substância, demonstrando a adequação do método a sua finalidade.

Na Tabela 3 são apresentados a recuperação, o desvio padrão relativo e o coeficiente de correlação para cada agrotóxico estudado através do tratamento estatístico da curva de calibração. Para todos os agrotóxicos estudados exceto o dicofol as recuperações na faixa de 70 a $120 \%$ para amostras de casca + polpa e para a polpa estão de acordo com o recomendado pelo CODEX ALIMENTARIUS [3].

O dicofol apresentou recuperação inferior a 70\%, porém após uma rastreabilidade de todo o procedimento envolvido verificou-se um problema de estabilidade com o padrão primário de dicofol (embora ele tenha apresentado uma taxa de recuperação melhor para o intervalo de tempo de fortificação de 4 horas). O processo de validação deverá ser repetido para este composto com um novo padrão, já que o dicofol tem seu uso permitido para citros no Brasil. O p,p'-DDE apresentou a recuperação entre 66 a $79 \%$ e o $\beta$-endosulfan entre 80 a $127 \%$

TABELA 3. Parâmetros estatísticos calculados para a laranja Lima (casca + polpa; polpa e casca).

\begin{tabular}{|c|c|c|c|c|c|c|c|c|c|}
\hline \multirow[b]{2}{*}{ Agrotóxico } & \multicolumn{3}{|c|}{ Casca + Polpa ${ }^{(a)}$} & \multicolumn{3}{|c|}{ Polpa ${ }^{\text {(a) }}$} & \multicolumn{3}{|c|}{ Casca $^{(a)}$} \\
\hline & $\begin{array}{c}\text { Coeficiente de } \\
\text { correlação } \\
(r)\end{array}$ & $\begin{array}{c}\text { Recuperação } \\
(\%)\end{array}$ & SR (\%) & $\begin{array}{l}\text { Coeficiente de } \\
\text { correlação } \\
(r)\end{array}$ & $\begin{array}{c}\text { Recuperação } \\
(\%)\end{array}$ & SR $(\%)$ & $\begin{array}{l}\text { Coeficiente de } \\
\text { correlação } \\
\text { (r) }\end{array}$ & $\begin{array}{c}\text { Recuperação } \\
(\%)\end{array}$ & SR $(\%)$ \\
\hline$\alpha-\mathrm{HCH}$ & 0,9949 & 78 a 116 & 2 a 11 & 0,9932 & 78 a 124 & 9 a 17 & 0,9947 & 81 a 122 & 3 a 22 \\
\hline HCB & 0,9900 & 70 a 88 & 2 a 6 & 0,9820 & 65 a 100 & 1 a 19 & 0,9879 & 86 a 123 & 4 a 8 \\
\hline$\beta-\mathrm{HCH}$ & 0,9927 & 76 a 117 & 2 a 15 & 0,9956 & 93 a 115 & 8 a 17 & 0,9869 & 73 a 116 & 4 a 11 \\
\hline$\gamma-\mathrm{HCH}$ & 0,9938 & 73 a 109 & 2 a 5 & 0,9952 & 81 a 125 & 8 a 16 & 0,9941 & 78 a 127 & 2 a 19 \\
\hline Heptacloro & 0,9929 & 89 a 112 & 5 a 15 & 0,9979 & 85 a 120 & 6 a 13 & 0,9918 & 106 a 136 & 2 a 10 \\
\hline Malation & 0,9965 & 84 a 91 & 9 a 18 & 0,9912 & 94 a 115 & 6 a 12 & 0,9994 & 91 a 100 & 6 a 19 \\
\hline Dicofol & 0,9914 & 56 a 60 & 8 a 16 & 0,9965 & 50 a 62 & 6 a 12 & 0,9983 & 53 a 61 & 8 a 11 \\
\hline HE & 0,9942 & 83 a 125 & 2 a 10 & 0,9969 & 96 a 110 & 10 a 16 & 0,9953 & 91 a 126 & 1 a 15 \\
\hline $0, p^{\prime}-\mathrm{DDE}$ & 0,9935 & 86 a 96 & 2 a 10 & 0,9956 & 105 a 128 & 10 a 15 & 0,9923 & 105 a 139 & 2 a 14 \\
\hline$\alpha$-endossulfan & 0,9959 & 83 a 84 & 2 a 6 & 0,9964 & 89 a 100 & 11 a 20 & 0,9935 & 96 a 123 & 2 a 8 \\
\hline$p, p^{\prime}-\mathrm{DDE}$ & 0,9966 & 66 a 79 & 2 a 6 & 0,9985 & 84 a 98 & 11 a 13 & 0,9969 & 76 a 89 & 1 a 16 \\
\hline Dieldrin & 0,9961 & 75 a 80 & 2 a 7 & 0,9962 & 90 a 102 & 11 a 16 & 0,9962 & 88 a 113 & 1 a 8 \\
\hline$\beta$-endossulfan & 0,9970 & 80 a 127 & 2 a 9 & 0,9964 & 100 a 113 & 11 a 15 & 0,9951 & 79 a 225 & 2 a 13 \\
\hline$p, p^{\prime}-\mathrm{DDD}$ & 0,9959 & 84 a 88 & 3 a 7 & 0,9973 & 105 a 118 & 11 a 16 & 0,9969 & 96 a 110 & 4 a 11 \\
\hline $0, p^{\prime}-\mathrm{DDT}$ & 0,9937 & 86 a 123 & 2 a 7 & 0,9962 & 101 a 120 & 9 a 20 & 0,9911 & 92 a 152 & 1 a 9 \\
\hline$p, p^{\prime}$-DDT & 0,9963 & 91 a 117 & 2 a 7 & 0,9978 & 103 a 113 & 9 a 16 & 0,9953 & 87 a 165 & 2 a 9 \\
\hline Metoxicloro & 0,9903 & 96 a 108 & 3 a 20 & 0,9951 & 107 a 116 & 9 a 16 & 0,9949 & 100 a 146 & 3 a 9 \\
\hline Diazinon & 0,9946 & 96 a 111 & 2 a 7 & 0,9904 & 89 a 119 & 8 a 15 & 0,9959 & 139 a 168 & 6 a 19 \\
\hline Fenitrotion & 0,9925 & 72 a 86 & 5 a 12 & 0,9971 & 83 a 95 & 7 a 13 & 0,9963 & 57 a 86 & 4 a 17 \\
\hline Clorotalonil & 0,9900 & 77 a 91 & 4 a 8 & 0,9956 & 92 a 112 & 7 a 15 & 0,9982 & 56 a 68 & 3 a 19 \\
\hline Permetrina-cis & 0,9918 & 89 a 102 & 5 a 23 & 0,9922 & 107 a 116 & 10 a 20 & 0,9859 & 114 a 145 & 7 a 19 \\
\hline Folpet & 0,9913 & 96 a 109 & 2 a 11 & 0,9991 & 104 a 128 & 8 a 17 & 0,9934 & 99 a 134 & 2 a 12 \\
\hline Metil-paration & 0,9971 & 97 a 104 & 5 a 15 & 0,9961 & 93 a 115 & 8 a 17 & 0,9873 & 81 a 104 & 6 a 21 \\
\hline Perm etrina-trans & 0,9949 & 88 a 98 & 5 a 24 & 0,9935 & 108 a 115 & 10 a 21 & 0,9795 & 132 a 203 & 6 a 25 \\
\hline Mirex & 0,9927 & 73 a 87 & 3 a 6 & 0,9923 & 95 a 113 & 10 a 18 & 0,9859 & 92 a 113 & 2 a 7 \\
\hline
\end{tabular}

(a) Dados obtidos para o intervalo de trabalho de 0,02 a $2,0 \mathrm{mg} / \mathrm{kg}$ para triplicatas de oito diferentes níveis de concentração estudado. 
para amostras da casca + polpa, entretanto essa pequena diferença foi irrelevante para os agrotóxicos em questão. No entanto, pode-se observar que nas análises realizadas somente com a casca o intervalo de recuperação na maioria dos agrotóxicos foi superior a 70 e $120 \%$, o que confirma a possibilidade dos mesmos ficarem retidos na casca.

Os desvios padrão relativos calculados também estão de acordo com o estabelecido pelo CODEX ALIMENTARIUS [3] que é de até 20\%. Nas análises de casca + polpa somente a permetrina apresentou resultado um pouco superior ao estabelecido, porém essa diferença foi insignificativa para o agrotóxico em questão.

Como a maior parte da laranja produzida no país está direcionada para a indústria de sucos, é de interesse do laboratório estender a pesquisa de resíduos de agrotóxicos em sucos de laranja a fim de se verificar a possivel contaminação do produto final, já que nem sempre as laranjas são lavadas antes da preparação do suco, seja pelo consumidor em sua residência ou em estabelecimentos comerciais, antes de sua extração em máquinas, uma vez que existe a possibilidade do suco ser contaminado por resíduos presentes nas cascas.

As taxas de recuperação foram obtidas através da média de duas determinações realizadas em paralelo para o nível de concentração estudado.

\section{4 - CONCLUSÕES}

Os resultados encontrados demonstram a habilidade do método estudado e são satisfatórios para as necessidades da rotina do laboratório, já que é um procedimento simples, de multi-resíduos, baixo custo e eficiente.

A presença da metidationa nas três amostras de laranja Lima orgânica nos leva a concluir que se deve incrementar a quantidade de agrotóxicos pesquisados em laranja, independentemente do uso permitido. Além disso, a pesquisa de amostras provenientes da agricultura orgânica deve ser levada em consideração já que não deveriam ser encontrados resíduos de agrotóxicos nesse tipo de cultivo.

\section{5 - REFERÊNCIAS BIBLIOGRÁFICAS}

[1] ARAÚJO, A.C.P.; TElles, D.L.; LIMA, L.L.A; RODRIGUES, J.; LIMA, T.L.A.; SILVA, M. Monitorização de Resíduos de Praguicidas em Frutas Destinadas à
Exportação. Revista Brasileira de Toxicologia, v. 14, n. 2, p. 43-48, 2001.

[2] ABECITRUS (Associação Brasileira dos Exportadores de Cítricos). [on-line]. 2003. Disponível: http:// www.abecitrus.com.br [capturado em 04 set. 2003].

[3] CODEX Alimentarius. Volume 2A. Pesticide Residues in Food. Methods of analysis and sampling. 2 ed., part 1, Roma. 2000.

[4] Determinação de resíduos de pesticidas em laranjas. In: Manual da Qualidade. Rio de Janeiro: INCQS/ FIOCRUZ. Seção 10 (65.3120.070).

[5] FAO (Food and Agricultural Organization). [on-line]. Disponivel: http://www.fao.org [capturado em 05 set. 2003].

[6] IBGE (Instituto Brasileiro de Geografia e Estatística). Levantamento Sistemático da Produção Agrícola [online]. 2003. Disponivel: http://www.ibge.gov.br [capturado em 04 set. 2003].

[7] MAPA (Ministério da Agricultura, Pecuária e Abastecimento). Fruticultura e Estatísticas Agrícolas. [online]. 2003. Disponível: http://www.agricultura.gov. br/fruticultura/ [capturado em 04 set. 2003].

[8] MDIC (Ministério do Desenvolvimento Indústria a Comércio Exterior). Cadeias produtivas. [on-line]. 2003. Disponivel: http://www.mdic.gov.br [capturado em 12 set. 2003].

[9] PARÂMETROS estatísticos para validação de resíduos de agrotóxicos em alimentos. In: Manual da Gualidade. Rio de Janeiro: INCQS/FIOCRUZ. Seção 10 (65.3120.081).

[10] BRASIL. Resolução-RE n 165, de 29 de agosto de 2003. Diretoria Colegiada da Agência Nacional de Vigilância Sanitária, do Ministério da Saúde. Diário Oficial [da República Federativa do Brasil], Brasília, 02 de setembro de 2003, p. 48-50, Seção 1.

[11] TORRES, C.M.; PICÓ, Y.; MAÑES, J. Comparison of octadecylsilica and graphitized carbon black as materials for solid - phase extraction of fungicide and insecticide residues from fruit and vegetables. Journal of Chromatography A, v. 778, p. 127-137, 1997.

[12] TORRES, C.M.; PICÓ, Y.; REDONDO, M.J.; MAÑES, J. Matrix solid - Phase dispersion extraction procedure for multiresidue pesticide analysis in Oranges. Journal of Chromatography A, v. 719, p. 95-103, 1996.

\section{6 - AGRADECIMENTOS}

Os autores agradecem a Dra. Adélia Cristina P. Araújo, ITEP-PE, pela contribuição nas análises por espectrometria de massas, realizadas neste estudo e à Fundação de Amparo a Pesquisa do Estado do Rio de Janeiro - FAPERJ, pela bolsa de Apoio Técnico I concedida a Tatiane dos Santos Neves; à UNESCO / ANVISA pela bolsa concedida a Maria Helena Wohlers Morelli Cardoso. 